\title{
Fundamental tone estimates for elliptic operators in divergence form and geometric applications
}

\section{GREGÓRIO P. BESSA ${ }^{1}$, LUQUÉSIO P. JORGE ${ }^{1}$, BARNABÉ P. LIMA ${ }^{2}$ and JOSÉ F. MONTENEGRO ${ }^{1}$}

\author{
${ }^{1}$ Universidade Federal do Ceará - UFC, Departamento de Matemática \\ Campus do Pici, 60455-760 Fortaleza, CE, Brasil \\ ${ }^{2}$ Universidade Federal do Piauí - UFPi, Departamento de Matemática \\ Campus Petrônio Portela, 64049-550 Teresina, PI, Brasil
}

\begin{abstract}
Manuscript received on October 31, 2005; accepted for publication on April 17, 2006;
\end{abstract} presented by MANFREDO DO CARMO

\begin{abstract}
We establish a method for giving lower bounds for the fundamental tone of elliptic operators in divergence form in terms of the divergence of vector fields. We then apply this method to the $L_{r}$ operator associated to immersed hypersurfaces with locally bounded $(r+1)$-th mean curvature $H_{r+1}$ of the space forms $\mathbb{N}^{n+1}(c)$ of constant sectional curvature $c$. As a corollary we give lower bounds for the extrinsic radius of closed hypersurfaces of $\mathbb{N}^{n+1}(c)$ with $H_{r+1}>0$ in terms of the $r$-th and $(r+1)$-th mean curvatures. Finally we observe that bounds for the Laplace eigenvalues essentially bound the eigenvalues of a self-adjoint elliptic differential operator in divergence form. This allows us to show that Cheeger's constant gives a lower bounds for the first nonzero $L_{r}$ eigenvalue of a closed hypersurface of $\mathbb{N}^{n+1}(c)$.
\end{abstract}

Key words: fundamental tone, $L_{r}$ operator, $r$-th mean curvature, extrinsic radius, Cheeger's constant.

\section{INTRODUCTION}

Let $\Omega$ be a domain in a smooth Riemannian manifold $M$ and let $\Phi: \Omega \rightarrow$ End $(T \Omega)$ be a smooth symmetric and positive definite section of the bundle of all endomorphisms of $T \Omega$. Each section $\Phi$ is associated to a second order self-adjoint elliptic operator $L_{\Phi}(f)=\operatorname{div}(\Phi \operatorname{grad} f), f \in C^{2}(\Omega)$. Observe that when $\Phi$ is the identity section then $L_{\Phi}=\triangle$, the Laplace operator. Recall that the 
$L_{\Phi}$-fundamental tone of $\Omega$ is given by

$$
\lambda^{L_{\Phi}}(\Omega)=\inf \left\{\frac{\int_{\Omega}\left|\Phi^{1 / 2} \operatorname{grad} f\right|^{2}}{\int_{\Omega} f^{2}} ; f \in C_{0}^{2}(\Omega) \backslash\{0\}\right\} .
$$

If $\Omega$ is bounded with smooth boundary $\partial \Omega \neq \emptyset$, the $L_{\Phi}$-fundamental tone of $\Omega$ coincides with the first eigenvalue $\lambda_{1}^{L_{\Phi}}(\Omega)$ of the Dirichlet eigenvalue problem $L_{\Phi} u+\lambda u=0$ on $\Omega$, with $u \mid \partial \Omega=0$, $u \in C^{2}(\Omega) \cap C^{0}(\bar{\Omega}) \backslash\{0\}$. If $\Omega$ is bounded with empty boundary $\partial \Omega=\emptyset$ then $\lambda^{L_{\Phi}}(\Omega)=0$.

A basic problem in Riemannian geometry is what lower and upper bounds for $\lambda^{L_{\Phi}}(\Omega)$ can be obtained in terms of Riemannian invariants. In this paper we show that the method established by Bessa and Montenegro (Bessa and Montenegro 2004) that gives lower bounds for the $\triangle$-fundamental tone can be extended for self-adjoint elliptic operators $L_{\Phi}$, (Theorem 2.1). Then we consider immersed hypersurfaces $\varphi: M \rightarrow \mathbb{N}^{n+1}(c)$ of the $(n+1)$-dimensional simply connected space form $\mathbb{N}^{n+1}(c)$ of constant sectional curvature $c \in\{1,0,-1\}$ with locally bounded $(r+1)$-th mean curvature such that the differential operators $L_{r}, r \in\{0,1, \ldots, n\}$ are elliptic. We give lower bounds for the $L_{r}$-fundamental tone of domains $\Omega \subset \varphi^{-1}\left(B_{\mathbb{N}^{n+1}(c)}(p, R)\right)$, in terms of the $r$-th and $(r+1)$-th mean curvatures $H_{r}, H_{r+1}$, (Theorem 3.2), where $B_{\mathbb{N}^{n+1}(c)}(p, R)$ is the geodesic ball of $\mathbb{N}^{n+1}(c)$ centered at $p$ with radius $R$. From these estimates we derive three geometric corollaries 3.4, 3.5 and 3.8 that should be viewed as an extension of a result of Jorge and Xavier (Jorge and Xavier 1981). It should be mentioned that these corollaries are related to results due to Vlachos (Vlachos 1997) and to Fontenele and Silva (Fontenele and Silva 2001), see Remark 3.7. In Theorem 3.10 we consider immersed hypersurfaces $M$ of $\mathbb{N}^{n+1}(c)$ such that the operators $L_{r}$ and $L_{s}$, $0 \leq r, s \leq n$ are elliptic and we compare the $L_{r}$ and $L_{s}$ fundamental tones $\lambda^{L_{r}}(\Omega), \lambda^{L_{s}}(\Omega)$ of domains $\Omega \subset M \subset \mathbb{N}^{n+1}(c)$. In section 4 we observe (Theorem 4.1) that in order to get bounds for the eigenvalues of a self-adjoint elliptic differential operator $L_{\Phi}$ we essentially need bounds for the Laplace operator eigenvalues. This allows us to use Cheeger's constant to give lower bounds for the first nonzero $L_{r}$-eigenvalue of a closed hypersurface of $\mathbb{N}^{n+1}(c)$. The results are stated and discussed in Sections 2, 3 and 4 and the proofs are given in Section 5.

\section{$L_{\Phi}$-FUNDAMENTAL TONE ESTIMATES}

Our main estimate is the following method for giving lower bounds for $L_{\Phi}$-fundamental tone of arbitrary domains of Riemannian manifolds. It extends the version of Barta's theorem (Barta 1937) proved by Cheng-Yau in (Cheng and Yau 1977). It is the same proof (with proper modifications) of a generalization of Barta's theorem proved in (Bessa and Montenegro 2004).

THEOREM 2.1. Let $\Omega$ be a domain in a Riemannian manifold and let $\Phi: \Omega \rightarrow$ End $(T \Omega)$ be a smooth symmetric and positive definite section of $\operatorname{End}(T \Omega)$. Then the $L_{\Phi}$-fundamental tone of $\Omega$ has the following lower bound

$$
\lambda^{L_{\Phi}}(\Omega) \geq \sup _{\mathcal{X}(\Omega)} \inf _{\Omega}\left[\operatorname{div}(\Phi X)-\left|\Phi^{1 / 2} X\right|^{2}\right]
$$


If $\Omega$ is bounded and with piecewise smooth boundary $\partial \Omega \neq \emptyset$ then we have equality in (2).

$$
\lambda^{L_{\Phi}}(\Omega)=\sup _{\mathcal{X}(\Omega)} \inf _{\Omega}\left[\operatorname{div}(\Phi X)-\left|\Phi^{1 / 2} X\right|^{2}\right]
$$

Where $\mathcal{X}(\Omega)$ is the set of all smooth vector fields on $\Omega$.

\section{GEOMETRIC APPLICATIONS}

Consider the linearized operator $L_{r}$ of the $(r+1)$-mean curvature

$$
H_{r+1}=\frac{S_{r+1}}{\left(\begin{array}{c}
n \\
r+1
\end{array}\right)}
$$

arising from normal variations of a hypersurface $M$ immersed into the $(n+1)$-dimensional simply connected space form $\mathbb{N}^{n+1}(c)$ of constant sectional curvature $c \in\{1,0,-1\}$, where $S_{r+1}$ is the $(r+1)$-th elementary symmetric function of the principal curvatures $k_{1}, k_{2}, \ldots, k_{n}$, see (Reilly 1973) and (Rosenberg 1993) for details. Recall that the elementary symmetric function of the principal curvatures are given by

$$
S_{0}=1, \quad S_{r}=\sum_{i_{1}<\cdots<i_{r}} k_{i_{1}} \cdots k_{i_{r}}, \quad 1 \leq r \leq n .
$$

Letting $A=-(\bar{\nabla} \eta)$ be the shape operator of $M$, where $\bar{\nabla}$ is the Levi-Civita connection of $\mathbb{N}^{n+1}(c)$ and $\eta$ a globally defined unit vector field normal to $M$, we can recursively define smooth symmetric sections $P_{r}: M \rightarrow$ End $(T M)$, for $r=0,1, \ldots, n$, called the Newton operators, setting $P_{0}=I$ and $P_{r}=S_{r} I d-A P_{r-1}$ so that $P_{r}(x): T_{x} M \rightarrow T_{x} M$ is a self-adjoint linear operator with the same eigenvectors as the shape operator $A$. The operator $L_{r}$ is the second order self-adjoint differential operator

$$
L_{P_{r}}(f)=\operatorname{div}\left(P_{r} \operatorname{grad} f\right)
$$

associated to the section $P_{r}$. However, the sections $P_{r}$ may be not positive definite and then the operators $L_{r}$ may not be elliptic. However, there are geometric hypothesis that imply the ellipticity of $L_{r}$, see for instance, Reilly 1973, Caffarelli et al. 1985, Korevaar 1988 or Barbosa and Colares 1997. Here we will not impose geometric conditions to guarantee ellipticity of the $L_{r}$, except in corollary 3.5. Instead we will ask the ellipticity on the set of hypothesis. It is known the ordered eigenvalues $\left\{\mu_{1}^{r}(x) \leq \ldots \leq \mu_{n}^{r}(x)\right\}$ of $P_{r}(x)$ depend continuously on $x \in M$. (Kato 1976 pages 106-109). In fact, this proof can be pushed to prove that they are Lipschitz thus differentiable almost everywhere. In addition, the respective eigenvectors $\left\{e_{1}(x), \ldots, e_{n}(x)\right\}$ form a smooth orthonormal frame in a neighborhood of every point. Set $\nu\left(P_{r}\right)=\sup _{x \in M}\left\{\mu_{n}^{r}(x)\right\}$ and $\mu\left(P_{r}\right)=\inf _{x \in M}\left\{\mu_{1}^{r}(x)\right\}$. Observe that if $\mu\left(P_{r}\right)>0$ then $P_{r}$ is positive definite, thus $L_{r}$ is elliptic. 
We need the following definition of locally bounded $(r+1)$-th mean curvature hypersurface in order to state our next result.

DEFINITION 3.1. An oriented immersed hypersurface $\varphi: M \hookrightarrow N$ of a Riemannian manifold $N$ is said to have locally bounded $(r+1)$-th mean curvature $H_{r+1}$ if for any $p \in N$ and $R>0$, the number

$$
h_{r+1}(p, R)=\sup \left\{\left|S_{r+1}(x)\right|=\left(\begin{array}{c}
n \\
r+1
\end{array}\right) \cdot\left|H_{r+1}(x)\right| ; x \in \varphi(M) \cap B_{N}(p, R)\right\}
$$

is finite. Here $B_{N}(p, R) \subset N$ is the geodesic ball of radius $R$ with center at $p \in N$.

Our next result generalizes in some aspects the main application of (Bessa and Montenegro 2003). There the first and fourth authors give lower bounds for $\triangle$-fundamental tone of domains in submanifolds with locally bounded mean curvature in complete Riemannian manifolds.

THEOREM 3.2. Let $\varphi: M \hookrightarrow \mathbb{N}^{n+1}(c)$ be an oriented hypersurface immersed with locally bounded $(r+1)$-th mean curvature $H_{r+1}$ for some $r \leq n-1$ and with $\mu\left(P_{r}\right)>0$. Let $B_{\mathbb{N}^{n+1}(c)}(p, R)$ be the geodesic ball centered at $p \in \mathbb{N}^{n+1}(c)$ with radius $R$ and $\Omega \subset \varphi^{-1}\left(\overline{B_{\mathbb{N}^{n+1}(c)}(p, R)}\right)$ be a connected component. Then the $L_{r}$-fundamental tone $\lambda^{L_{r}}(\Omega)$ of $\Omega$ has the following lower bounds.

i. For $c=1$ and $0<R<\cot ^{-1}\left[\frac{(r+1) \cdot h_{r+1}(p, R)}{(n-r) \cdot \inf _{\Omega} S_{r}}\right]$ we have that

$$
\lambda^{L_{r}}(\Omega) \geq 2 \cdot \frac{1}{R}\left[(n-r) \cdot \cot [R] \cdot \inf _{\Omega} S_{r}-(r+1) \cdot h_{r+1}(p, R)\right] .
$$

ii. For $c \leq 0, h_{r+1}(p, R) \neq 0$ and $0<R<\frac{(n-r) \cdot \inf _{\Omega} S_{r}}{(r+1) \cdot h_{r+1}(p, R)}$ we have that

$$
\lambda^{L_{r}}(\Omega) \geq 2 \cdot \frac{1}{R^{2}}\left[(n-r) \cdot \inf _{\Omega} S_{r}-(r+1) \cdot R \cdot h_{r+1}(p, R)\right] .
$$

iii. If $c \leq 0, h_{r+1}(p, R)=0$ and $R>0$ we have that

$$
\lambda^{L_{r}}(\Omega) \geq \frac{2(n-r) \inf _{\Omega} S_{r}}{R^{2}}
$$

DEFINITION 3.3. Let $\varphi: M \hookrightarrow N$ be an isometric immersion of a closed Riemannian manifold into a complete Riemannian manifold $N$. For each $x \in N$, let $r(x)=\sup _{y \in M} \operatorname{dist}_{N}(x, \varphi(y))$. The extrinsic radius $R_{e}(M)$ of $M$ is defined by

$$
R_{e}(M)=\inf _{x \in N} r(x)
$$

Moreover, there is a point $x_{0} \in N$ called the barycenter of $\varphi(M)$ in $N$ such that $R_{e}(M)=r\left(x_{0}\right)$. 
COROLlaRY 3.4. Let $\varphi: M \hookrightarrow B_{\mathbb{N}^{n+1}(c)}(R) \subset \mathbb{N}^{n+1}(c)$ be a complete oriented hypersurface with bounded $(r+1)$-th mean curvature $H_{r+1}$ for some $r \leq n-1, R$ chosen as in Theorem (3.2). Suppose that $\mu\left(P_{r}\right)>0$ so that the $L_{r}$ operator is elliptic. Then $M$ is not closed.

Corollary 3.5. Let $\varphi: M \hookrightarrow \mathbb{N}^{n+1}(c)^{1}, c \in\{1,0,-1\}$ be an oriented closed hypersurface with $H_{r+1}>0$. Then there is an explicit constant $\Lambda_{r}=\Lambda_{r}\left(c, \inf _{M} S_{r}, \sup _{M} S_{r+1}\right)>0$ such that the extrinsic radius $R_{e}(M) \geq \Lambda_{r}$.

i. For $c=1, \Lambda_{r}=\cot ^{-1}\left[\frac{(r+1) \cdot \sup _{M} S_{r+1}}{(n-r) \cdot \inf _{M} S_{r}}\right]$.

ii. For $c \in\{0,-1\}, \Lambda_{r}=\frac{(n-r) \cdot \inf _{M} S_{r}}{(r+1) \cdot \sup _{M} S_{r+1}}$.

REMARK 3.6. The hypothesis $H_{r+1}>0$ implies that $H_{j}>0$ and $L_{j}$ are elliptic for $j=0,1, \ldots r$, see Barbosa and Colares 1997, Caffarelli et al. 1985 or Korevaar 1988. Thus in fact have that $R_{e} \geq \max \left\{\Lambda_{0}, \cdots, \Lambda_{r}\right\}$.

REMARK 3.7. Jorge and Xavier, (Jorge and Xavier 1981) proved the inequalities of Corollary 3.5 when $r=0$ for complete submanifolds with scalar curvature bounded from below contained in a compact ball of a complete Riemannian manifold. Moreover, for $c=-1$ their inequality is slightly better. It is possible to give sharp estimates for the extrinsic radius of a closed hypersurface of $\mathbb{N}^{n+1}(c)$ in terms of $\sup _{M}\left|H_{r}\right|$ alone. Vlachos (Vlachos 1997) proved a result that implies that, for each

$$
\begin{aligned}
1 \leq r \leq n, & R_{e}(M) \geq\left(\sup \left|H_{r}\right|\right)^{-1 / r}, \\
R_{e}(M) & \geq \cot ^{-1}\left(\sup \left|H_{r}\right|\right)^{1 / r} \\
R_{e}(M) & \geq \operatorname{coth}^{-1}\left(\sup \left|H_{r}\right|\right)^{1 / r}
\end{aligned}
$$

if $c=0, c=1$ or $c=-1$ respectively, and that in any case the equality holds if and only if $M$ is a geodesic sphere of the ambient space. The result of Vlachos was extended to any ambient space by Fontenele and Silva (Fontenele and Silva 2001).

REMARK 3.8. An interesting question is: Is it true that any closed oriented hypersurface with $\mu_{1}^{r}(M)>0$ and $H_{r+1}=0$ intersect every great circle? For $r=0$ it is true and it was proved by T. Frankel, (Frankel 1966).

We now consider immersed hypersurfaces $\varphi: M \hookrightarrow \mathbb{N}^{n+1}(c)$ with $L_{r}$ and $L_{s}$ elliptic. We can compare the $L_{r}$ and $L_{s}$ fundamental tones of a domain $\Omega \subset M$. In particular we can compare with its $L_{0}$-fundamental tone.

THEOREM 3.9. Let $\varphi: M \hookrightarrow \mathbb{N}^{n+1}(c)$ be an oriented $n$-dimensional hypersurface $M$ immersed into the $(n+1)$-dimensional simply connected space form of constant sectional curvature $c$ and

${ }^{1}$ If $c=1$ suppose that $\mathbb{N}^{n+1}(c)$ is the open hemisphere of $\mathbb{S}_{+}^{n+1}$. 
$\mu\left(P_{r}\right)>0$ and $\mu\left(P_{s}\right)>0,0 \leq s, r \leq n-1$. Let $\Omega \subset M$ be a domain with compact closure and piecewise smooth nonempty boundary. Then the $L_{r}$ and $L_{s}$ fundamental tones satisfies the following inequalities

$$
\lambda^{L_{r}}(\Omega) \geq \frac{\mu\left(P_{r}\right)}{v\left(P_{s}\right)} \cdot \lambda^{L_{s}}(\Omega)
$$

From (9) we have in particular that

$$
v\left(P_{r}\right) \cdot \lambda^{\triangle}(\Omega) \geq \lambda^{L_{r}}(\Omega) \geq \mu\left(P_{r}\right) \cdot \lambda^{\triangle}(\Omega)
$$

\section{CLOSED EIGENVALUE PROBLEM}

Let $M$ be a closed hypersurface of a simply connected space form $\mathbb{N}^{n+1}(c)$. The interesting problem is what bounds can one obtain for the first nonzero $L_{r}$-eigenvalue $\lambda_{1}^{L_{r}}(M)$ in terms of the geometries of $M$ and of the ambient space. Upper bounds for the first nonzero $\triangle$-eigenvalue or even for the first nonzero $L_{r}$-eigenvalue, $r \geq 1$ have been obtained by many authors in contrast with lower bounds that are rare. For instance, Reilly (Reilly 1977) extending earlier result of Bleecker and Weiner (Bleecker and Weiner 1976) obtained upper bounds for $\lambda_{1}^{\Delta}(M)$ of a closed submanifold $M$ of $\mathbb{R}^{m}$ in terms of the total mean curvature of $M$. Reilly's result applied to compact submanifolds of the sphere $M \subset \mathbb{S}^{m+1}(1)$, this latter viewed as a hypersurface of the Euclidean space $\mathbb{S}^{m+1}(1) \subset \mathbb{R}^{m+2}$ obtains upper bounds for $\lambda_{1}^{\triangle}(M)$, see Alencar, Do Carmo and Rosenberg in Alencar et al. 1993. Heintze (Heintze 1988) extended Reilly's result to compact manifolds and Hadamard manifolds $\bar{M}$. In particular for the hyperbolic space $\mathbb{H}^{n+1}$. The best upper bounds for the first nonzero $\triangle$-eigenvalue of closed hypersurfaces $M$ of $\mathbb{H}^{n+1}$ in terms of the total mean curvature of $M$ was obtained by El Soufi and Ilias (Soufi and Ilias 1992). Regarding the $L_{r}$ operators, Alencar, Do Carmo and Rosenberg (Alencar et al. 1993) obtained sharp (extrinsic) upper bound the first nonzero eigenvalue $\lambda_{1}^{L_{r}}(M)$ of the linearized operator $L_{r}$ of compact hypersurfaces $M$ of $\mathbb{R}^{m+1}$ with $S_{r+1}>0$. Upper bounds for $\lambda_{1}^{L_{r}}(M)$ of compact hypersurfaces of $\mathbb{S}^{n+1}, \mathbb{H}^{n+1}$ under the hypothesis that $L_{r}$ is elliptic were obtained by Alencar, Do Carmo, Marques in (Alencar et al. 2001) and by Alias and Malacarne in (Alias and Malacarne 2004) see also the work of Veeravalli (Veeravalli 2001). On the other hand, lower bounds for $\lambda_{1}^{L_{r}}(M)$ of closed hypersurfaces $M \subset$ $\mathbb{N}^{n+1}(c)$ are not so well studied as the upper bounds, except for $r=0$ in which case $L_{0}=\triangle$. In this paper we make a simple observation (Theorem 4.1) that to obtain lower and upper bounds for the $L_{\Phi}$-eigenvalues (Dirichlet or Closed eigenvalue problem) it is enough to obtain lower and upper bounds for the eigenvalues of $\Phi$ and for the eigenvalues for the Laplacian in the respective problem. When applied to the $L_{r}$ operators (supposing them elliptic) we obtain lower bounds for closed hypersurfaces of the space forms via Cheeger's lower bounds for the first $\triangle$-eigenvalue of closed manifolds. Let $\left\{\mu_{1}(x) \leq \ldots \leq \mu_{n}(x)\right\}$ be the ordered eigenvalues of $\Phi(x)$. Setting $v(\Phi)=\sup _{x \in \Omega}\left\{\mu_{n}(x)\right\}$ and $\mu(\Phi)=\inf _{x \in \Omega}\left\{\mu_{1}(x)\right\}$ we have the following theorem. 
THEOREM 4.1. Let $\lambda^{L_{\Phi}}(\Omega)$ denote the $L_{\Phi}$-fundamental tone of $\Omega$ if $\Omega$ is unbounded or $\partial \Omega \neq \emptyset$ and the first nonzero $L_{\Phi}$-eigenvalue $\lambda_{1}^{L_{\Phi}}(\Omega)$ if $\Omega$ is a closed manifold. Then $\lambda^{L_{\Phi}}(\Omega)$ satisfies the following inequalities,

$$
v(\Phi) \cdot \lambda^{\triangle}(\Omega) \geq \lambda^{L_{\Phi}}(\Omega) \geq \mu(\Phi) \cdot \lambda^{\triangle}(\Omega),
$$

where $\lambda^{\Delta}(\Omega)$ is the $\triangle$-fundamental tone of $\Omega$ or the first nonzero $\triangle$-eigenvalue of $\Omega$.

Let $M$ be a closed $n$-dimensional Riemannian manifold, Cheeger (Cheeger 1970) defined the following constant given by

$$
h(M)=\inf _{S} \frac{\operatorname{vol}_{n-1}(S)}{\min \left\{\operatorname{vol}_{n}\left(\Omega_{1}\right), \operatorname{vol}_{n}\left(\Omega_{2}\right)\right\}},
$$

where $S \subset M$ ranges over all connected closed hypersurfaces dividing $M$ in two connected components, i.e. $M=\Omega_{1} \cup \Omega_{2}, \Omega_{1} \cap \Omega_{2}=\emptyset$ such that $S=\partial \Omega_{1}=\partial \Omega_{2}$ and he proved that the first nonzero $\triangle$-eigenvalue $\lambda_{1}^{\triangle}(M) \geq h(M)^{2} / 4$.

COROLlARY 4.2. Let $\varphi: M \hookrightarrow \mathbb{N}^{n+1}(c), c \in\{1,0,-1\}^{2}$ be an oriented closed hypersurface with $H_{r+1}>0$. Then the first nonzero $L_{r}$-eigenvalue of $M$ has the following lower bound

$$
\lambda_{1}^{L_{r}}(M) \geq \mu\left(P_{r}\right) \cdot \frac{h^{2}(M)}{4}
$$

\section{PROOF OF THE RESULTS}

\section{PROOF OF THEOREM 2.1.}

Let $\Omega$ be an arbitrary domain, $X$ be a smooth vector field on $\Omega$ and $f \in C_{0}^{\infty}(\Omega)$. The vector field $f^{2} \Phi X$ has compact support supp $\left(f^{2} \Phi X\right) \subset \operatorname{supp}(f) \subset \Omega$. Let $\mathcal{S}$ be a regular domain containing the support of $f$. We have by the divergence theorem that

$$
\begin{aligned}
0=\int_{\mathcal{S}} \operatorname{div}\left(f^{2} \Phi X\right) & =\int_{\Omega} \operatorname{div}\left(f^{2} \Phi X\right) \\
& =\int_{\Omega}\left[\left\langle\operatorname{grad} f^{2}, \Phi X\right\rangle+f^{2} \operatorname{div}(\Phi X)\right] \\
& \geq \int_{\Omega}\left[-2 \cdot|f| \cdot\left|\Phi^{1 / 2} \operatorname{grad} f\right| \cdot\left|\Phi^{1 / 2} X\right|+\operatorname{div}(\Phi X) \cdot f^{2}\right] \\
& \geq \int_{\Omega}\left[-\left|\Phi^{1 / 2} \operatorname{grad} f\right|^{2}-f^{2} \cdot\left|\Phi^{1 / 2} X\right|^{2}+\operatorname{div}(\Phi X) \cdot f^{2}\right] .
\end{aligned}
$$

Therefore

$$
\begin{aligned}
\int_{\Omega}\left|\Phi^{1 / 2} \operatorname{grad} f\right|^{2} & \geq \int_{\Omega}\left[\operatorname{div}(\Phi X)-\left|\Phi^{1 / 2} X\right|^{2}\right] f^{2} \\
& \geq \inf _{\Omega}\left[\operatorname{div}(\Phi X)-\left|\Phi^{1 / 2} X\right|^{2}\right] \int_{\Omega} f^{2}
\end{aligned}
$$

\footnotetext{
${ }^{2}$ If $c=1$ suppose that $\mathbb{N}^{n+1}(c)$ is the open hemisphere of $\mathbb{S}_{+}^{n+1}$.
} 
By the variational formulation (1) of $\lambda^{L_{\Phi}}(\Omega)$ this inequality above implies that

$$
\lambda^{L_{\Phi}}(\Omega) \geq \inf _{\Omega}\left[\operatorname{div}(\Phi X)-\left|\Phi^{1 / 2} X\right|^{2}\right]
$$

When $\Omega$ is a bounded domain with smooth boundary $\partial \Omega \neq \emptyset$ then $\lambda^{L_{\Phi}}(\Omega)=\lambda_{1}^{L_{\Phi}}(\Omega)$. This proof above shows that

$$
\lambda_{1}^{L_{\Phi}}(\Omega) \geq \inf _{\Omega}\left[\operatorname{div}(\Phi X)-\left|\Phi^{1 / 2} X\right|^{2}\right] .
$$

Let $v \in C^{2}(\Omega) \cap C^{0}(\bar{\Omega})$ be a positive first $L_{\Phi^{-}}$-eigenfunction ${ }^{3}$ of $\Omega$ and if we set $X_{0}=-\operatorname{grad} \log (v)$ we have that

$$
\begin{aligned}
& \operatorname{div}\left(\Phi X_{0}\right)-\left|\Phi^{1 / 2} X_{0}\right|^{2}=-\operatorname{div}((1 / v) \Phi \operatorname{grad} v)-\left(1 / v^{2}\right)\left|\Phi^{1 / 2} \operatorname{grad} v\right|^{2} \\
& \quad=\left(1 / v^{2}\right)\langle\operatorname{grad} v, \Phi \operatorname{grad} v\rangle-(1 / v) \operatorname{div}(\Phi \operatorname{grad} v)-\left(1 / v^{2}\right)\left|\Phi^{1 / 2} \operatorname{grad} v\right|^{2} \\
& \quad=-(1 / v) \operatorname{div}(\Phi \operatorname{grad} v)=-L_{\Phi}(v) / v=\lambda_{1}^{L_{\Phi}}(\Omega) .
\end{aligned}
$$

This proves (3).

\section{Proof of TheOREM 3.2 AND COROLlaries $3.4,3.5$ AND 3.8}

We start this section stating few lemmas necessary to construct the proof of Theorem 3.2. The first lemma was proved in (Jorge and Koutrofiotis 1980) for the Laplace operator and for the $L_{r}$ operator in (Lima 2000). We reproduce its proof to make the exposition complete.

LEMMA 5.1. Let $\varphi: M \hookrightarrow \mathbb{N}^{n+1}(c)$ be a hypersurface immersed in $(n+1)$-dimensional simply connected space form $\mathbb{N}^{n+1}(c)$ of constant sectional curvature c. Let $g: \mathbb{N}^{n+1}(c) \rightarrow \mathbb{R}$ be a smooth function and set $f=g \circ \varphi$. Identify $X \in T_{p} M$ with $d \varphi(p) X \in T_{\varphi(p)} \varphi(M)$ then we have that

$$
L_{r} f(p)=\sum_{i=1}^{n} \mu_{i}^{r} \operatorname{Hess} g(\varphi(p))\left(e_{i}, e_{i}\right)+\operatorname{Trace}\left(A P_{r}\right)\langle\operatorname{grad} g, \eta\rangle
$$

PROOF. Each $P_{r}$ is also associated to a second order self-adjoint differential operator defined by $\square f=$ Trace ( $P_{r}$ Hess $(f)$ ) see (Cheng and Yau 1977, Hartmann 1978). We have that

$$
\square f=\operatorname{Trace}\left(P_{r} \operatorname{Hess}(f)\right)=\operatorname{div}\left(P_{r} \operatorname{grad} f\right)-\left\langle\text { Trace }\left(\nabla P_{r}\right), \operatorname{grad} f\right\rangle .
$$

Rosenberg (Rosenberg 1993) proved that when the ambient manifold is the simply connected space form $\mathbb{N}^{n+1}(c)$ then Trace $\left(\nabla P_{r}\right)$ grad $\equiv 0$, see also (Reilly 1973). Thus one has that $L_{r} f=$ Trace $\left(P_{r}\right.$ Hess $\left.(f)\right)$. Using Gauss equation to compute Hess $(f)$ we obtain

$$
\text { Hess } f(p)(X, Y)=\text { Hess } g(\varphi(p))(X, Y)+\langle\operatorname{grad} g, \alpha(X, Y)\rangle_{\varphi(p)},
$$

where $\langle\alpha(X, Y), \eta\rangle=\langle A(X), Y\rangle$. Let $\left\{e_{i}\right\}$ be an orthonormal frame around $p$ that diagonalize the section $P_{r}$ so that $P_{r}(x)\left(e_{i}\right)=\mu_{i}^{r}(x) e_{i}$. Thus

$$
L_{r} f=\sum_{i=1}^{n}\left\langle P_{r} \operatorname{Hess} f\left(e_{i}\right), e_{i}\right\rangle=\sum_{i=1}^{n}\left\langle\operatorname{Hess} f\left(e_{i}\right), \mu_{i}^{r} e_{i}\right\rangle=\sum_{i=1}^{n} \mu_{i}^{r} \operatorname{Hess} f\left(e_{i}, e_{i}\right)
$$

$3 v \in C^{2}(\Omega) \cap H_{1}^{0}(\Omega)$ if $\partial \Omega$ is not smooth. 
Substituting (19) into (20) we have that

$$
\begin{aligned}
L_{r} f & =\sum_{i=1}^{n} \mu_{i}^{r} \operatorname{Hess} g\left(e_{i}, e_{i}\right)+\left\langle\operatorname{grad} g, \sum_{i=1}^{n} \mu_{i}^{r} \alpha\left(e_{i}, e_{i}\right)\right\rangle \\
& =\sum_{i=1}^{n} \mu_{i}^{r} \operatorname{Hess} g\left(e_{i}, e_{i}\right)+\left\langle\operatorname{grad} g, \alpha\left(\sum_{i=1}^{n} P_{r}\left(e_{i}\right), e_{i}\right)\right\rangle \\
& =\sum_{i=1}^{n} \mu_{i}^{r} \operatorname{Hess} g\left(e_{i}, e_{i}\right)+\operatorname{Trace}\left(A P_{r}\right)\langle\operatorname{grad} g, \eta\rangle
\end{aligned}
$$

Here Hess $f(X)=\nabla_{X} \operatorname{grad} f$ and Hess $f(X, Y)=\left\langle\nabla_{X} \operatorname{grad} f, Y\right\rangle$. The next two lemmas we are gong to present are well known and their proofs are easily found in the literature thus we will omit them here.

Lemma 5.2 [Hessian Comparison Theorem]. Let $M$ be a complete Riemannian manifold and $x_{0}, x_{1} \in M$. Let $\gamma:\left[0, \rho\left(x_{1}\right)\right] \rightarrow M$ be a minimizing geodesic joining $x_{0}$ and $x_{1}$ where $\rho(x)$ is the distance function dist ${ }_{M}\left(x_{0}, x\right)$. Let $K$ be the sectional curvatures of $M$ and $v(\rho)$, defined below.

$$
v(\rho)=\left\{\begin{array}{cll}
k_{1} \cdot \operatorname{coth}\left(k_{1} \cdot \rho(x)\right), & \text { if } & \sup _{\gamma} K=-k_{1}^{2} \\
\frac{1}{\rho(x)}, & \text { if } & \sup _{\gamma} K=0 \\
k_{1} \cdot \cot \left(k_{1} \cdot \rho(x)\right), & \text { if } & \sup _{\gamma} K=k_{1}^{2} \text { and } \rho<\pi / 2 k_{1} .
\end{array}\right.
$$

Let $X=X^{\perp}+X^{T} \in T_{x} M, X^{T}=\left\langle X, \gamma^{\prime}\right\rangle \gamma^{\prime}$ and $\left\langle X^{\perp}, \gamma^{\prime}\right\rangle=0$. Then

$$
\text { Hess } \rho(x)(X, X)=\operatorname{Hess} \rho(x)\left(X^{\perp}, X^{\perp}\right) \geq v(\rho(x)) \cdot\left\|X^{\perp}\right\|^{2}
$$

See (Schoen and Yau 1994) for a proof.

LEMMA 5.3. Let $p \in M$ and $1 \leq r \leq n-1$, let $\left\{e_{i}\right\}$ be an orthonormal basis of $T_{p} M$ such that $P_{r}\left(e_{i}\right)=\mu_{i}^{r} e_{i}$ and $A\left(e_{i}\right)=k_{i} e_{i}$. Then

i. $\operatorname{Trace}\left(P_{r}\right)=\sum_{i=1}^{n} \mu_{i}^{r}=(n-r) S_{r}$

ii. Trace $\left(A P_{r}\right)=\sum_{i=1}^{n} k_{i} \mu_{i}^{r}=(r+1) S_{r+1}$

In particular, if the Newton operator $P_{r}$ is positive definite then $S_{r}>0$.

To prove Theorem (3.2) set $g: B(p, R) \subset \mathbb{N}^{n+1}(c) \rightarrow \mathbb{R}$ given by $g=R^{2}-\rho^{2}$, where $\rho$ is the distance function $(\rho(x)=\operatorname{dist}(x, p))$ of $\mathbb{N}^{n+1}(c)$. Setting $f=g \circ \varphi$ we obtain by (17) that

$$
L_{r} f=\sum_{i=1}^{n} \mu_{i}^{r} \cdot \operatorname{Hess} g\left(e_{i}, e_{i}\right)+(r+1) \cdot S_{r+1} \cdot\langle\operatorname{grad} g, \eta\rangle,
$$

since Trace $\left(A P_{r}\right)=(r+1) \cdot S_{r+1}$. Letting $X=-\operatorname{grad} \log f$ we have that

$$
\operatorname{div} P_{r} X-\left|P_{r}^{1 / 2} X\right|^{2}=-L_{r}(f) / f
$$


then by Theorem (2.1) we have that

$$
\begin{aligned}
\lambda^{L_{r}}(\Omega) & \geq \inf _{\Omega}\left(-L_{r} f / f\right) \\
& =\inf _{\Omega}\left\{-\frac{1}{g}\left[\sum_{i=1}^{n} \mu_{i}^{r} \cdot \operatorname{Hess} g\left(e_{i}, e_{i}\right)+(r+1) \cdot S_{r+1} \cdot\langle\operatorname{grad} g, \eta\rangle\right]\right\} .
\end{aligned}
$$

Computing the Hessian of $g$ we have that

$$
\begin{aligned}
\text { Hess } g\left(e_{i}, e_{i}\right) & =\left\langle\nabla_{e_{i}} \operatorname{grad} g, e_{i}\right\rangle \\
& =-2\left\langle\nabla_{e_{i}} \rho \operatorname{grad} \rho, e_{i}\right\rangle \\
& =-2\left\langle\operatorname{grad} \rho, e_{i}\right\rangle^{2}-2 \rho\left\langle\nabla_{e_{i}} \operatorname{grad} \rho, e_{i}\right\rangle \\
& =-2\left\langle\operatorname{grad} \rho, e_{i}\right\rangle^{2}-2 \rho \operatorname{Hess} \rho\left(e_{i}, e_{i}\right) .
\end{aligned}
$$

Therefore we have that

$$
-\frac{L_{r} f}{f}=\frac{2}{R^{2}-\rho^{2}}\left[\sum_{i=1}^{n} \mu_{i}^{r}\left[\left\langle\operatorname{grad} \rho, e_{i}\right\rangle^{2}+\rho \operatorname{Hess} \rho\left(e_{i}, e_{i}\right)\right]+(r+1) \cdot S_{r+1} \cdot \rho \cdot\langle\operatorname{grad} \rho, \eta\rangle\right]
$$

Setting $e_{i}^{T}=\left\langle\operatorname{grad} \rho, e_{i}\right\rangle \operatorname{grad} \rho$ and $e_{i}^{\perp}=e_{i}-e_{i}^{T}$, by the Hessian Comparison Theorem we have that

$$
\sum_{i=1}^{n} \mu_{i}^{r}\left[\left\langle\operatorname{grad} \rho, e_{i}\right\rangle^{2}+\rho \operatorname{Hess} \rho\left(e_{i}, e_{i}\right)\right] \geq \sum_{i=1}^{n} \mu_{i}^{r}\left[\left\|e_{i}^{T}\right\|^{2}+\rho \cdot v(\rho)\left\|e_{i}^{\perp}\right\|^{2}\right]
$$

and

$$
(r+1) \cdot S_{r+1} \cdot \rho \cdot\langle\operatorname{grad} \rho, \eta\rangle \leq(r+1) R \cdot h_{r+1}(p, R)
$$

From (28) and (29) we have that

$$
\begin{aligned}
\lambda^{L_{r}}(\Omega) & \geq \inf _{\Omega}\left(-L_{r} f / f\right) \\
& \geq 2 \cdot \inf _{\Omega}\left\{\frac{1}{R^{2}-\rho^{2}}\left[\sum_{i=1}^{n} \mu_{i}^{r}\left[\left\|e_{i}^{T}\right\|^{2}+\rho \cdot v(\rho)\left\|e_{i}^{\perp}\right\|^{2}\right]-(r+1) \cdot R \cdot h_{r+1}(p, R)\right]\right\}
\end{aligned}
$$

If $c \leq 0$ then $\rho \cdot v(\rho) \geq 1$ thus from (30) we have that

$$
\begin{aligned}
\lambda^{L_{r}}(\Omega) & \geq 2 \cdot \frac{1}{R^{2}}\left[\inf _{\Omega}\left\{\sum_{i=1}^{n} \mu_{i}^{r}\left[\left\|e_{i}^{T}\right\|^{2}+\left\|e_{i}^{\perp}\right\|^{2}\right]\right\}-(r+1) \cdot R \cdot h_{r+1}(p, R)\right] \\
& =2 \cdot \frac{1}{R^{2}}\left[\inf _{\Omega} \sum_{i=1}^{n} \mu_{i}^{r}-(r+1) \cdot R \cdot h_{r+1}(p, R)\right] \\
& =2 \cdot \frac{1}{R^{2}}\left[(n-r) \inf _{\Omega} S_{r}-(r+1) \cdot R \cdot h_{r+1}(p, R)\right] .
\end{aligned}
$$


If $c>0$ then $\rho \cdot v(\rho)=\rho \cdot \sqrt{c} \cdot \cot [\sqrt{c} \rho] \leq 1$ thus from (30) we have that

$$
\begin{aligned}
\lambda^{L_{r}}(\Omega) & \geq 2 \cdot \frac{1}{R^{2}}\left[\inf _{\Omega}\left\{\sum_{i=1}^{n} \mu_{i}^{r}\left[\left\|e_{i}^{T}\right\|^{2}+\left\|e_{i}^{\perp}\right\|^{2}\right] \rho \cdot \sqrt{c} \cdot \cot [\sqrt{c} \rho]\right\}-(r+1) \cdot R \cdot h_{r+1}(p, R)\right] \\
& =2 \cdot \frac{1}{R^{2}}\left[\inf _{\Omega}\left\{\sum_{i=1}^{n} \mu_{i}^{r} \rho \sqrt{c} \cot [\sqrt{c} \rho]\right\}-(r+1) \cdot R \cdot h_{r+1}(p, R)\right] \\
& =2 \cdot \frac{1}{R^{2}}\left[(n-r) \cdot R \cdot \sqrt{c} \cdot \cot [\sqrt{c} R] \cdot \inf _{\Omega} S_{r}-(r+1) \cdot R \cdot h_{r+1}(p, R)\right] .
\end{aligned}
$$

To prove the Corollaries (3.4) and (3.5) observe that the hypotheses $\mu\left(P_{r}\right)(M)>0$ (in Corollary 3.4) and $H_{r+1}>0$ (in Corollary 3.5) imply that the $L_{r}$ is elliptic. If the immersion is bounded (contained in a ball of radius $R$, for those choices of $R$ ) and $M$ is closed we would have by one hand that the $L_{r}$-fundamental tone would be zero and by Theorem (3.2) that it would be positive. Then $M$ can not be closed if the immersion is bounded. On the other hand if $M$ is closed a ball of radius $R$ centered at the barycenter of $M$ could not contain $M$ because the fundamental tone estimates for any connected component $\Omega \subset \varphi^{-1}\left(\varphi(M) \cap B_{\mathbb{N}^{n+1}(c)}(p, R)\right.$ is positive. Showing that $M \neq \Omega$.

\section{PROOF OF THEOREM 3.9.}

Let $\varphi: W \hookrightarrow \mathbb{N}^{n+1}(c)$ be an isometric immersion of an oriented $n$-dimensional Riemannian manifold $W$ into a $(n+1)$-dimensional simply connected space form of sectional curvature $c$. Let $M \subset W$ be a domain with compact closure and piecewise smooth nonempty boundary and suppose that the Newton operators $P_{r}$ and $P_{s}, 0 \leq s, r \leq n-1$ are positive definite when restricted to $M$. Given a vector field $X$ on $M$ we can find a vector field $Y$ on $M$ such that $P_{r} X=\kappa \cdot P_{S} Y, \kappa$ constant. Now

$$
\begin{aligned}
\operatorname{div}\left(P_{r} X\right)-\left|P_{r}^{1 / 2} X\right|^{2} & =\kappa \cdot \operatorname{div}\left(P_{s} Y\right)-\left\langle P_{r} X, X\right\rangle \\
& =\kappa \cdot \operatorname{div}\left(P_{s} Y\right)-\kappa^{2}\left\langle P_{s} Y, P_{r}^{-1} P_{s} Y\right\rangle \\
& =\kappa \cdot\left[\operatorname{div}\left(P_{s} Y\right)-\left|P_{s}^{1 / 2} Y\right|^{2}+\left|P_{s}^{1 / 2} Y\right|^{2}-\kappa \cdot\left|P_{r}^{-1 / 2} P_{s} Y\right|^{2}\right]
\end{aligned}
$$

Consider $\left\{e_{i}\right\}$ be an orthonormal basis such that $P_{r} e_{i}=\mu_{i}^{r} e_{i}$ and $P_{s} e_{i}=\mu_{i}^{s} e_{i}$. Letting $Y=$ $\sum_{i=1}^{n} y_{i} e_{i}$ then

$$
\begin{aligned}
\left|P_{s}^{1 / 2} Y\right|^{2}-\kappa \cdot\left|P_{r}^{-1 / 2} P_{s} Y\right|^{2} & =\sum_{i=1}^{n} \mu_{i}^{s} y_{i}^{2}-\kappa \sum_{i=1}^{n} \frac{\left(\mu_{i}^{s}\right)^{2}}{\mu_{i}^{r}} y_{i}^{2} \\
& =\sum_{i=1}^{n} \mu_{i}^{s} y_{i}^{2}\left[1-\kappa \cdot \frac{\mu_{i}^{s}}{\mu_{i}^{r}}\right] \\
& \geq 0, \text { if } \kappa \leq \frac{\mu\left(P_{r}\right)}{v\left(P_{s}\right)}
\end{aligned}
$$


Combining (33) with (34) and by Theorem (2.1) we have that

$$
\begin{aligned}
\lambda^{L_{r}}(M) & =\sup _{X} \inf _{M} \operatorname{div}\left(P_{r} X\right)-\left|P_{r}^{1 / 2} X\right|^{2} \\
& \geq \kappa \cdot \sup _{Y} \inf _{M} \operatorname{div}\left(P_{s} Y\right)-\left|P_{s}^{1 / 2} Y\right|^{2} \\
& =\kappa \cdot \lambda^{L_{s}}(M),
\end{aligned}
$$

for every $0<\kappa \leq \frac{\mu\left(P_{r}\right)}{v\left(P_{s}\right)}$. This proves (9).

\section{PROOF OF THEOREM 4.1 .}

Recall that for any smooth symmetric section $\Phi: \Omega \rightarrow$ End $(T \Omega)$ there is an open and dense subset $U \subset \Omega$ where the ordered eigenvalues $\left\{\mu_{1}(x) \leq \ldots \leq \mu_{n}(x)\right\}$ of $\Phi(x)$ depend continuously in all $\Omega$. In addition, the respective eigenvectors $\left\{e_{1}(x), \ldots, e_{n}(x)\right\}$ form a smooth orthonormal frame in a neighborhood of every point of $\Omega$, see (Kato 1976). Let $f \in C_{0}^{2}(\Omega) \backslash\{0\}\left(f \in C^{2}(\Omega)\right.$ with $\int_{\Omega} f=0$ ) be an admissible function for (the closed $L_{\Phi}$-eigenvalue problem if $\Omega$ is a closed manifold) the Dirichlet $L_{\Phi}$-eigenvalue problem. It is clear that $f$ is an admissible function for the respective $\triangle$-eigenvalue problem. Writing grad $f(x)=\sum_{i=1}^{n} e_{i}(f) e_{i}(x)$ we have that

$$
\begin{aligned}
\left|\Phi^{1 / 2} \operatorname{grad} f\right|^{2}(x) & =\langle\Phi \operatorname{grad} f, \operatorname{grad} f\rangle(x) \\
& =\left\langle\sum_{i=1}^{n} \mu_{i}(x) e_{i}(f) e_{i}, \sum_{i=1}^{n} e_{i}(f) e_{i}\right\rangle \\
& =\sum_{i=1}^{n} \mu_{i}(x) e_{i}(f)^{2}(x) .
\end{aligned}
$$

From (36) we have that

$$
v(\Phi) \cdot|\operatorname{grad} f|^{2}(x) \geq\left|\Phi^{1 / 2} \operatorname{grad} f\right|^{2}(x) \geq \mu(\Phi) \cdot|\operatorname{grad} f|^{2}(x)
$$

and

$$
v(\Phi) \cdot \frac{\int_{M}|\operatorname{grad} f|^{2}}{\int_{M} f^{2}} \geq \frac{\int_{M}\left|\Phi^{1 / 2} \operatorname{grad} f\right|^{2}}{\int_{M} f^{2}} \geq \mu(\Phi) \cdot \frac{\int_{M}|\operatorname{grad} f|^{2}}{\int_{M} f^{2}}
$$

Taking the infimum over all admissible functions in (38) we obtain (11).

\section{RESUMO}

Estabelecemos um método para obter limites inferiores para o tom fundamental de operadores elípticos em forma divergente em termos do divergente de campos de vetores. Aplicamos esse método para os operadores $L_{r}$ associados a hipersuperfícies imersas nas formas espaciais $\mathbb{N}^{n}(c)$ de curvatura seccional constante $c$ com $(r+1)$-curvatura média $H_{r+1}$ localmente limitada. Obtemos como corolário limites inferiores para o raio extrínseco de hipersuperfícies compactas das formas espaciais $\mathbb{N}^{n}(c)$ com $H_{r+1}>0$ em termos das $r$-ésima 
e $r+1$-ésima curvatura médias. Finalmente, observamos que limites para os autovalores do Laplaciano essencialmente limitam os autovalores dos operadores elípticos em forma divergente. Isso permite mostrar que a constante de Cheeger limita inferiormente o primeiro autovalor não-nulo dos operadores $L_{r}$ em hypersuperfícies compactas de $\mathbb{N}^{n}(c)$.

Palavras-chave: tom fundamental, operador $L_{r}, r$-curvatura média, raio extrínseco, constante de Cheeger.

\section{REFERENCES}

Alencar H, do Carmo M and Rosenberg H. 1993. On the first eigenvalue of the linearized operator of the $r$-th mean curvature of a hypersurface. Ann Global Anal Geom 11: 387-395.

Alencar H, do Carmo M and Marques FC. 2001. Upper bounds for the first eigenvalue of the operator $\mathrm{Lr}$ and some applications. Illinois J of Math 45: 851-863.

Alias L and Malacarne M. 2004. Sharp upper bounds for the first positive eigenvalue of the linearized operator of the higher order mean curvature of closed hypersurfaces into a Riemannian space form. Illinois J Math 48: 219-240.

Barbosa JL And Colares AG. 1997. Stability of hypersurfaces with constant $r$-mean curvature. Ann Global Anal Geom 15: 277-297.

BARTA J. 1937. Sur la vibration fundamentale d'une membrane. C R Acad Sci 204: 472-473.

Bessa GP And Montenegro JF. 2003. Eigenvalue estimates for submanifolds with locally bounded mean curvature. Ann Global Anal Geom 24: 279-290.

Bessa GP And Montenegro JF. 2004. An Extension of Barta's Theorem and Geometric Applications. ArchivX Paper Id: math.DG/0308099.

BLEECKER D AND WeINER J. 1976. Extrinsic bounds for $\lambda_{1}$ of $\Delta$ on a compact manifold. Comment Math Helv 51: 601-609.

Caffarelli L, Nirenberg L And Spruck J. 1985. The Dirichlet problem for nonlinear second order elliptic equations III. Functions of the eigenvalues of the Hessian. Acta Math 155: 261-301.

Cheeger J. 1970. A lower bound for the smallest eigenvalue of the Laplacian Problems in Analysis. Princenton University Press, Princenton, New Jersey, p. 195-199.

Cheng SY AND YAU ST. 1977. Hypersurfacces with Constant Scalar Curvature. Math Ann 225: 195-204.

FontenEle F AND SiLVA S. 2001. A tangency principle and applications. Illinois J of Math 45: 213-228.

FRANKEL T. 1966. On the fundamental group of a compact minimal submanifold. Ann of Math 83: 68-73.

HARTMANN P. 1978. On complete hypersurfaces of nonnegative sectional curvatures and constant $\mathrm{m}^{\text {th }}$ mean curvature. Trans AMS 245: 363-374.

Heintze E. 1988. Extrinsic upper bound for $\lambda_{1}$. Math Ann 280: 389-402.

Jorge LP AND Koutrofiotis D. 1980. An estimate for the curvature of bounded submanifolds. Amer J of Math 103: 711-725. 
JORGE LP AND XAVIER F. 1981. An inequality between the exterior diameter and the mean curvature of bounded immersions. Math Z 178: 77-82.

KATO T. 1976. Perturbation Theory for Linear Operators. Grundlehren der mathematischen Wissenschaften, Springer-Verlag 132: 122-124.

KorevaAR N. 1988. Sphere theorems via Alexandrov for constant Weingarten curvature hypersurfaces. Appendix to a Note of A. Ros. J Diff Geom 27: 221-223.

Lima BP. 2000. Omori-Yau Maximum Principle for the Operator $L_{r}$ and its Applications, Doctoral Thesis, Universidade Federal do Ceará-UFC.

REILLY R. 1973. Variational properties of functions of the mean curvatures for hypersurfaces in space forms. J Differential Geom 8: 465-477.

REILLY R. 1977. On the first eigenvalue of the Laplacian for compact submanifolds of the Euclidean space. Comment Math Helv 52: 525-533.

Rosenberg H. 1993. Hypersurface of Constant curvatures in space forms. Bull Sc Math 117: 211-239.

SOUfI AE AND ILIAS S. 1992. Une inegalite du type "Reilly" pour les sous-varietes de l'espace hyperbolique. Comm Math Helv 67: 167-181.

Schoen R AND YAU ST. 1994. Lectures on Differential Geometry. Conference Proceedings and Lecture Notes. In: Geometry and Topology 1.

VEERAVALI A. 2001. On the first Laplacian eigenvalue and the center of gravity of compact hypersurfaces. Comment Math Helv 76: 155-160.

VlachOS T. 1997. A characterization for geodesic spheres in space forms. Geom Dedicata 68: 73-78. 